PAPER

\title{
Short latency responses in the averaged electro-oculogram elicited by vibrational impulse stimuli applied to the skull: could they reflect vestibulo-ocular reflex function?
}

\section{P Jombík, V Bahýl}

J Neurol Neurosurg Psychiatry 2005;76:222-228. doi: 10.1136/jnnp.2003.027615

See end of article for authors' affiliations

Correspondence to: P Jombík, Hosp. Zvolen, Department of Neurology, Lab Clin Neurophysiol, 96089 Zvden, SK; bahyl@ vsld.tuzvo.sk

Received

5 September 2003

In revised form

21 April 2004

Accepted 8 June 2004 whether vibrational impulse stimuli applied to the skull can be used to evoke the Objectives: To investigate whether vibrational impulse stimulic
vestibulo-ocular reflex (VOR) and detect vestibular lesions.

Methods: Twenty four patients with unilateral vestibular loss (UVD), five with bilateral vestibular loss, two with ocular palsies, and 10 healthy subjects participated. Vibrations of the skull were induced with head taps and with a single period of $160 \mathrm{~Hz}$ tone burst on the inion, vertex, and the mastoids while the patients viewed a distant target. Several patients were also examined while viewing a near target, with eccentric gaze and in tilted postures. Responses were recorded by EOG.

Results: Responses occurred between $5 \mathrm{~ms}$ and $20 \mathrm{~ms}$ and seemed to be compensatory to the second phase of the sine wave of vibration impulse and were greatly diminished/absent in patients with bilateral VD and ocular palsies. The patients with UVD had asymmetrical responses in the vertical EOG with stimuli applied on the inion and vertex, with enhancement of the response amplitude on the side of vestibular loss and/or diminution on the healthy side. The asymmetry ratios between the healthy subjects and patients with UVD, and among patients with UVD were statistically significant. Some gaze and positional influences could be demonstrated consistent with otolithic reflexes.

Conclusion: If the asymmetric responses to skull vibration in UVD result from passive oscillatory movements of the orbital tissues they may reflect the otolith mediated sustained skew torsion. Conversely, if generated by active eye movements, their likely origin is a phasic VOR.
B oth primary otolithic and canal afferents of a monkey can be activated by vibration. The lowest phase locking thresholds have been determined at -70 to $-80 \mathrm{~dB}$ and median values in the most sensitive frequency range (200$400 \mathrm{~Hz}$ ) at -20 to $-40 \mathrm{~dB}$ of gravitational acceleration. ${ }^{1}$ It is still not clear whether activation of vestibular receptors by vibration has the same mechanical basis as the response to more physiological head movements. Mechanical factors are not the only determinants of response dynamics since vestibular nerve fibres can show a frequency dependent increase in gain greater than that predicted for the mechanics of sensory end organs. ${ }^{12}$

The mechanics of the otolithic membrane can be approximated by a damped second-order system with a resonant frequency of the order of 50-500 Hz. Thus, in contrast to the cupula-endolymph system in which the upper frequency limit is set below $60 \mathrm{~Hz}$, the otolithic membrane is much better suited for transmission of bone vibrations in the audio frequency range. Conversely, canal neurones tend to be more irregular than otolith neurones, and hence might be expected to have lower vibration thresholds.

The primary function of the vestibulo-ocular reflexes (VOR) is to provide short latency compensatory eye movements early in the movement, before visual tracking comes into play. Since both the angular VOR (aVOR) and compensatory linear VOR (IVOR) operate with high pass characteristics relative to head velocity input, both might respond to skull vibrations. Both aVOR and IVOR are modulated by viewing distance, or more precisely by ocular vergence. However, this dependence is much stronger for lVOR. ${ }^{34}$ Moreover, the naso-occipital lVOR is also strongly influenced by gaze eccentricities relative to the naso-occipital axis. ${ }^{56}$ Latency of the human aVOR is approximately $10 \mathrm{~ms}$ with only slight intersubject variation (range 6-15 ms), coexistent with a three neurone arc. ${ }^{78}$ The default gain of the human aVOR at onset, elicited by low and modest accelerations is appropriate for distant targets. ${ }^{8}$ Higher accelerations activate responses without delay, for the expression of the effects produced by distance.' Disynaptic connections between otoliths and oculomotor neurones have been demonstrated in cats, and studies in monkeys have found the lVOR latency to be similar to that of the aVOR. ${ }^{6}{ }^{10}{ }^{11}$ The mean latency of human IVOR is approximately $30 \mathrm{~ms}$ with high intersubject variability and gain dependent on the target distance from the onset..$^{12} 13$ However, similar to the aVOR, the latency of the lVOR can be very brief in some subjects, and hence a disynaptic neurone arc may operate in humans as well. ${ }^{13}$ It has been suggested that fast projections might carry a baseline IVOR signal independent of viewing distance. ${ }^{14}$ Moreover, it is possible that compensatory IVOR uses pathways in common with the aVOR. ${ }^{15}$ The dynamics of the lVOR dramatically enhance the responses to frequencies of linear accelerations above $0.5 \mathrm{~Hz}$ independent of viewing distance. ${ }^{15-17}$ In contrast with compensatory lVOR, the orienting IVOR codes orientation of the eyes in space. Its purpose is to produce responses to static or low frequency tilts of the head relative to constant gravitational force. However, the oculomotor responses required to compensate tilt and translation differ. Unlike the translational compensatory IVOR, there is no geometric requirement for tilt responses to be modulated by changes in fixation distance. The orienting lVOR operates with low pass dynamics, and contrary to transient ocular movements generated by compensatory lVOR and aVOR, it

Abbreviations: a/IVOR, angular/linear vestibulo-ocular reflexes; BVD, bilateral vestibular loss; CFA, craniofacial asymmetries; EOG, electrooculography; UVD, unilateral vestibular dysfunction VCR, vestibulo-collic reflex 
induces appropriate eye rotations and/or torsions that tend to be more sustained. ${ }^{15}{ }^{17}$

Experimental and clinical data suggest that vibrational stimuli can evoke vestibulospinal and possibly also vestibuloocular responses. ${ }^{18-24}$ Thus short pulses of vibration may be appropriate for recording the earliest part of the VOR, provided that mechanical artefacts and other cranial reflexes do not obscure these responses. ${ }^{25}$ By applying vibrational stimuli to the skull, most of the mechanical energy dissipates at the bony interface and the acceleration transmitted to bone is attenuated by $\sim 20 \mathrm{~dB} .^{26}$ Vibrations are then conducted through bone with only minor losses $<5 \mathrm{~dB} .{ }^{27}$ The velocity of propagation is estimated to be $260 \mathrm{~m} / \mathrm{s}^{28}$

In a preliminary study skull vibrations were generated by head taps or by sound stimuli conducted in bone. Head accelerations generated by stimulation along the nasooccipital (x) axis were measured by an accelerometer attached to the forehead. The maximal accelerations were about $0.55 \mathrm{~cm} / \mathrm{s}^{2}$ and $0.25 \mathrm{~cm} / \mathrm{s}^{2}$ for head taps and $160 \mathrm{~Hz}$ bone conducted tone bursts, respectively. The stimuli elicited transient responses with short latencies in the averaged electro-oculography (EOG) that may be compatible with a disynaptic VOR. If the recorded events were generated solely by horizontal or vertical eye rotations, the amplitudes of eye movements would approach $\sim 1-4^{\circ}$ in the responses elicited by head taps and $0.25-1^{\circ}$ in the bone conducted tone bursts. The responses in vertical EOG were generally symmetrical in normal subjects. However, sometimes surprisingly asymmetrical responses were found in otherwise normal subjects with craniofacial asymmetries (CFA). The reason may be the different orientation of vestibular end organs on the left and right sides. ${ }^{29}$ Moreover, owing to asymmetry of skull thickness the sensitivity of the labyrinths to vibrations may be different. There was a tendency for diminution of the responses in older subjects, and there were no responses in cadavers.

The purpose of the current study was to investigate the utility of head taps and bone conducted short tone bursts as an effective means to test the function of the VOR.

\section{METHODS \\ Patients}

Two groups of patients with unilateral vestibular dysfunction (UVD) were investigated. The first group comprised 10 patients with total unilateral vestibular deafferentation due to surgery for an acoustic neuroma. There was no evidence of significant brainstem or cerebellar disorder. They were tested within five weeks to 12 years of surgery with a mean interval of 45 months. Five were also investigated before the surgery. The second, more heterogeneous, group comprised 14 patients with UVD of various causes with consistent abnormality of the vestibulo-collic reflex (VCR) elicited with head taps. Five patients with profound bilateral vestibular loss (BVD) were also investigated. Finally, a patient with unilateral third nerve palsy, and another with advanced stage of progressive external ophthalmoplegia were also tested. The control group comprised 10 normal volunteers without a history or clinical signs of vestibular or ocular motor disorder or visible CFA. A requirement for normal results of a VCR elicited with taps were added to the clinical inclusion criteria to reduce the likelihood of the bias from CFA. The study was performed in accordance with the 1964 Declaration of Helsinki protocol and with approval of the institutional ethics committee.

\section{Test procedures}

The subjects were seated upright in a dimly lit room, and viewed a small illuminated target directly ahead at a distance of $3.5 \mathrm{~m}$. Several subjects were also examined viewing near targets at distances of $25 \mathrm{~cm}$ and/or $15 \mathrm{~cm}$ in eccentric horizontal and vertical gaze positions, as well as lying supine and prone and with eyes closed. Care was taken to ensure that the subjects' jaw muscles were relaxed. Skull taps were applied approximately at a rate of one to two per second. The reflex hammer was fitted with an inertial switch that produced a delay of 2-3 ms, so the latencies of the recorded responses were apparently shortened by the same amount. Bone conducted sound stimuli consisted of single period of $160 \mathrm{~Hz}$ logon (which is a particular type of tone burst with a raised cosine, instead of trapezoidal enveloping function) and were delivered by a clinical bone vibrator with a repetition rate of three per second. All patients were investigated using the first method. The second method was used only in the first UVD group (that is, operated patients). Stimuli were applied on the inion, the vertex, and both the mastoids, thereby acceleration of skull was caused along three mutually orthogonal axes, the naso-occipital (x), dorsoventral (z), and interaural $(y)$. However, due to the route of spread of the vibration waves to the labyrinths, the stimulation was really orthogonal only for the stimuli along the naso-occipital and interaural axes. Responses were recorded by means of $\mathrm{Ag} /$ $\mathrm{AgCl}$ surface electrodes producing bilateral monocular vertical and binocular horizontal EOG. The signal was amplified and band pass filtered $(5-2000 \mathrm{~Hz}), 2 \times 30-60$ responses for head taps and $2 \times 200$ responses for bone conducted tone bursts were averaged, using a sampling rate of $10000 \mathrm{~Hz}$ for each channel $5 \mathrm{~ms}$ before and $45 \mathrm{~ms}$ after each stimulus. The upward deflection of the recorded eye position signal in vertical and horizontal EOG channels corresponded to upward and rightward eye movement respectively. The VCR elicited with taps was recorded in all healthy subjects and in patients with vestibular disorders according to the method described by Halmagyi et al. ${ }^{18}$

\section{Data analysis}

EOG is feasible for accurate recording of horizontal eye rotations only. Measured potentials associated with vertical eye movements are distorted because the upper eyelid does not maintain a constant position with respect to the cornea. Moreover, the negative pole of the corneoretinal dipole does not lie exactly at the fovea, but instead it is displaced nasally by about $15^{\circ}$. This means that the electrical field is not precisely aligned with the optic axis, thus torsional movements of the globe may give rise to potential changes, that are misinterpreted as horizontal or vertical movements. ${ }^{30}{ }^{31}$ Due to the complexity of the recorded signals and the limited reliability of our recording technique, detailed quantitative analysis was avoided, with the exception of inter-side amplitude differences of EOG potentials in vertical channels with stimulation along the $\mathrm{x}$ axis.

\section{RESULTS}

\section{Basic characteristics of the responses}

Biphasic or triphasic responses were recorded 5-20 ms after stimulus onset. Onsets occurred between $5 \mathrm{~ms}$ and $15 \mathrm{~ms}$, and peaks before $20 \mathrm{~ms}$ (figs $1-3$ ). These responses were absent in all EOG records of the patient with progressive external ophthalmoplegia and in two of the five patients with $\mathrm{BVD}$, and were greatly diminished in the other three patients with BVD. Responses were also absent ipsilaterally in the vertical EOG records of the patient with third nerve palsy (fig 4). In horizontal EOG records elicited by tapping on the mastoids there was a small passive response with a zero latency culminating in the first $5 \mathrm{~ms}$. Following the stimulus generated by the bone vibrator, an electrical artefact comprised the initial parts of the records up to $9 \mathrm{~ms}$. Otherwise, regardless of the mode of elicitation the responses were similar in latency and shape. 


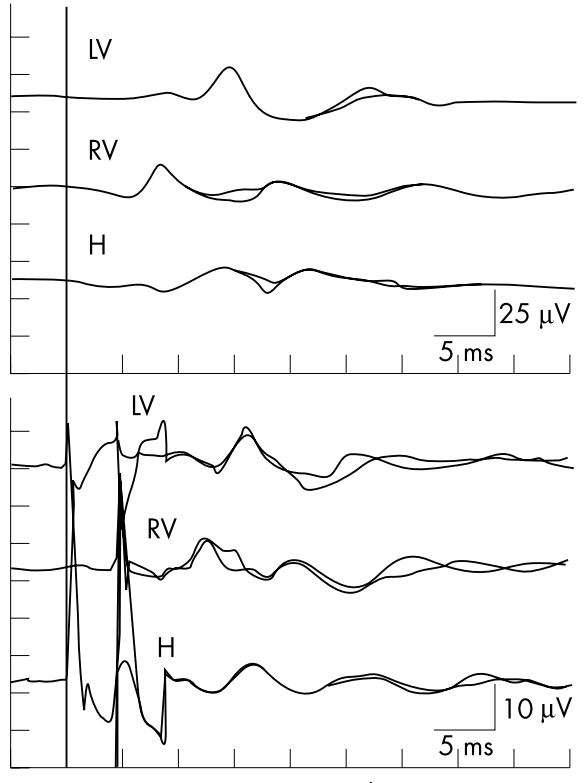

Stim L Mastoid

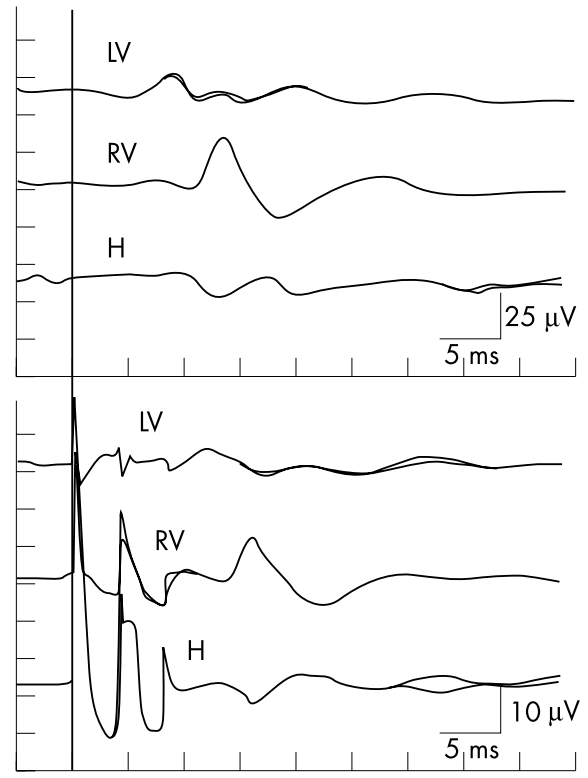

Stim R Mastoid
Figure 1 Averaged electrooculography (EOG) responses in the right and left mastoids in a normal subject elicited by stimuli along the interaural axis. Top panels: head taps elicited responses; bottom panels: $160 \mathrm{~Hz}$ bone conducted sound. All panels: traces from top to bottom correspond to the left and right vertical and horizontal binocular EOG channels, respectively; vertical lines indicate the timing of the stimuli. In horizontal EOG records elicited by tapping a small passive response with a zero latency decayed before the main responses appeared. Following the stimulus generated by the bone vibrator, an electrical artefact comprised the initial parts of the records up to $9 \mathrm{~ms}$. The signal averaged $5 \mathrm{~ms}$ before and $45 \mathrm{~ms}$ after the stimulus and records of two averaged trials for each condition were superimposed. The main biphasic or triphasic responses were apparently compensatory in horizontal but disconjugate in vertical EOG records; the direction reversed with oppositely directed stimuli. Note that responses elicited by head taps and bone conducted sound were almost identical.
Responses elicited by stimuli along the interaural $(y)$ axis

In the binocular horizontal EOG channel the main deflection of the trace was rightward directed with stimuli applied to the left mastoid and vice versa. The responses of the left and right eyes in the vertical EOG channels were disconjugate. The amplitude of the response on the ipsilateral side of the stimulation was larger than on the contralateral side and the peak latencies of the left and right eyes were also different. However, responses were mirror images of each other, with a reversal in direction when comparing responses elicited from the left versus the right side (fig 1). These symmetrical relations were lost in patients with UVD, due to a decrease of the amplitude on the healthy side and direction/latency shifts (fig 2).

Responses elicited by stimuli along the nasooccipital ( $x$ ) and dorsoventral $(z)$ axes

The stimuli elicited symmetrical biphasic or triphasic responses with a dominant upwards directed component in the vertical EOG channels only. Responses were symmetrical in normal subjects, but asymmetrical with enhanced amplitudes on the paretic and/or decreased amplitudes on the healthy side in patients with UVD (fig 3). Inter-side amplitude difference was statistically significant for both subgroups of patients with UVD (fig 5). This asymmetry was

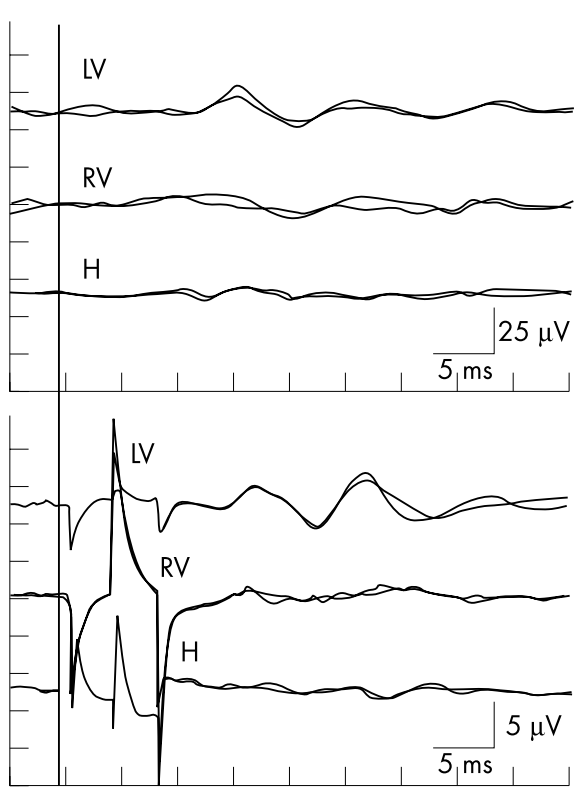

Stim L Mastoid

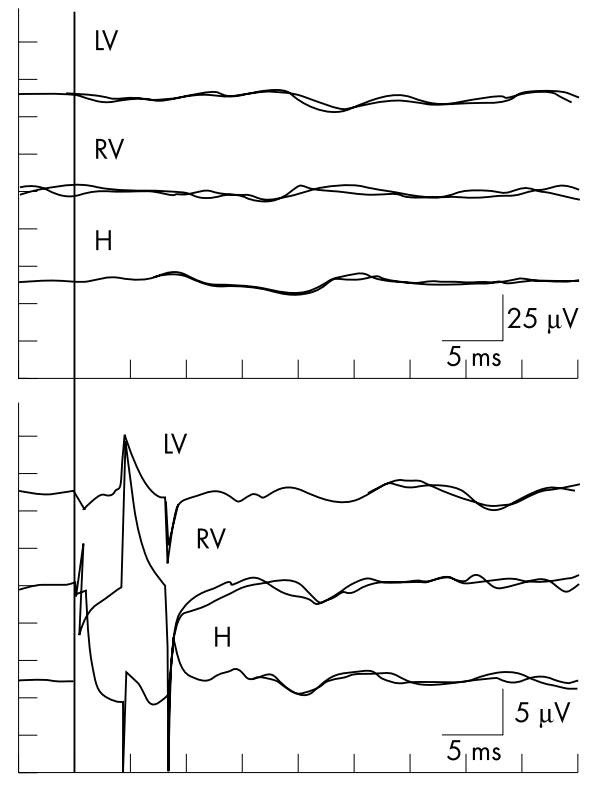

Stim R Mastoid
Figure 2 Averaged EOG responses elicited with stimuli along the interaural (y) axis. This figure represents the records of a patient with unilateral vestibular dysfunction affer surgery for an acoustic neuroma on the left side. See legend of fig 1 for details of the EOG channels, the timing of the stimulus and the time-base. The panels show the responses to both kinds of stimuli applied to left and right mastoids in the same manner as in fig 1.

Amplitudes of the responses in vertical EOG channels were larger when stimuli were applied to the lesioned side and the reversal in direction by oppositely directed stimuli was incomplete. 

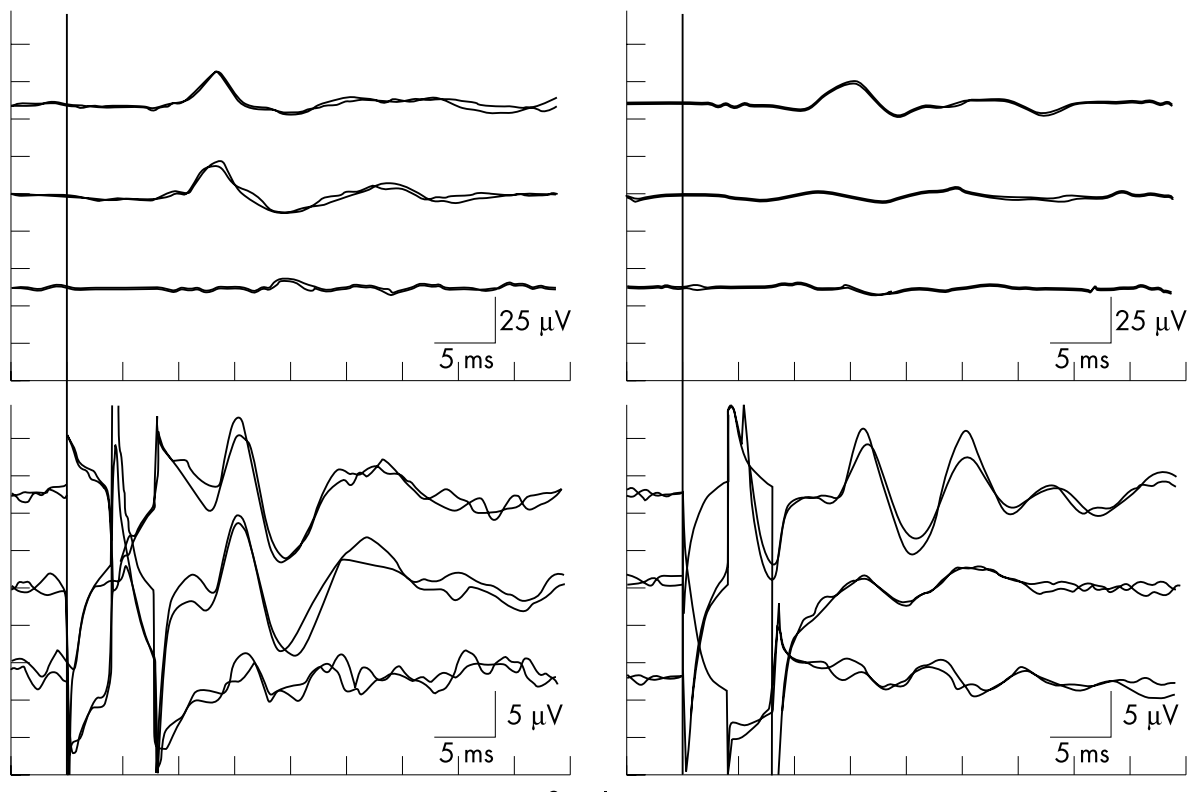

Figure 3 Averaged EOG responses elicited with stimuli along the nasooccipital $(x)$ axis Top panels show responses to head taps and bottom panels to bone conducted sound. See legend of fig 1 for details of the EOG channels, the timing of the stimulus and the time-base. The left panels show the responses of the same normal subject as in fig 1. The right panels show responses of the same patient with unilateral vestibular dysfunction after surgery for an acoustic neuroma on the left side as in fig 2. Significant biphasic and triphasic responses were elicited only in vertical EOG channels and were almost identical regardless of the type of stimulus. Responses were symmetrical in the normal subjects but asymmetrical with enhancement on the lesioned and/or diminution on the healthy side in patients with unilateral vestibular dysfunction.

Stim Inion

already present even in those patients examined before surgery. However, the probability of an idiosyncratic asymmetry of responses in these patients was low since they all had neuroma of moderate size $(20-30 \mathrm{~mm})$ with some vestibular loss before surgery. Moreover, the probability of an incidental occurrence of the abovementioned asymmetry in the group of surgically treated patients based on categorical data analysis was only $0.5 \%$.

\section{Kinematic considerations and positional effects}

Responses were similar whether the eyes were closed or open. Inconsistent findings were obtained with fixation of a near target: the responses were unchanged or even showed decreased amplitudes. Gaze influences were studied in responses elicited from stimuli along the naso-occipital $(\mathrm{x})$ axis. Upward gaze increased whereas downward gaze decreased the amplitude of responses in the vertical EOG channels. Horizontal gaze increased the amplitude on the side of the adducting and decreased it on the side of the abducting eye in healthy subjects as well as patient with UVD. Stimulation during horizontal gaze deviations also generated reverse directed responses in the horizontal EOG channel-that is, responses that were oppositely directed with regard to gaze direction. Moreover, amplitudes were decreased in vertical

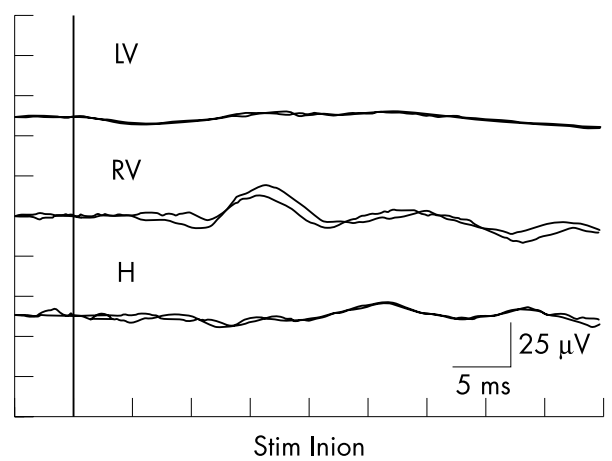

Figure 4 Averaged EOG responses elicited with head taps on the inion in the patient with a third nerve palsy on the left side. The responses in the left vertical EOG channel are absent. See legend of fig 1 for details of assembly of the EOG channels.
EOG channels bilaterally with stimulation along the nasooccipital $(\mathrm{x})$ axis in supine and prone positions.

Healthy subjects had a normal VCR bilaterally, whereas it was attenuated or absent ipsilaterally in all patients with UVD and bilaterally in all patients with BVD.

\section{DISCUSSION}

Vestibular response amplitudes (up to 100\%), elicited by vibrations can have even in normal subjects, considerable inter-side differences and the amplitude difference can approach $100 \%{ }^{20}$ This may be due to CFA, which can be invisible to the naked eye, or to hidden remnants of past vestibular insult. Thus the findings of the current study with regard to the control group as well as the second heterogeneous UVD group with unspecified vestibular pathology might be biased due to the selection criteria based on the findings of the VCR elicited by head taps. However, the findings in the first UVD group with anatomically proved complete vestibular deafferentation were undoubtedly consequences of the vestibular disorder.

\section{Passive oscillatory responses and lid artefacts}

To determine the origin of the recorded events the passive movements of electrodes, eyelids, and the globe should be taken into account. The natural frequency of oscillation for orbital tissues is above $12 \mathrm{~Hz}$ with a resonance frequency in the range of 50-63 Hz, so vibrations applied to the skull could induce passive eye and lid movements. ${ }^{832}{ }^{33}$ The amplitudes of the responses were in the range of values measured in vibration induced passive eye movements. ${ }^{34}$ Tonic innervation and ageing may change the viscoelastic properties of the eye muscles and modulate the resonance frequency and amplitude. Thus the absence or diminution of the responses in cadavers as well as individuals with palsies of the extraocular muscles and BVD could not exclude the possibility of passive oscillatory origin of responses. Indeed all but one of the currently presented patients with BVD were of advanced age.

Vertical EOG records always reflect an interaction of the eye and lid movement. The eyelid acts as sliding electrode, increasingly shunting the positive corneal pole to the upper EOG electrode while covering the larger surface of the cornea, or moving downward.$^{30}$ In all vertical eye movements, the lids follow the globes closely but small differences between lid 


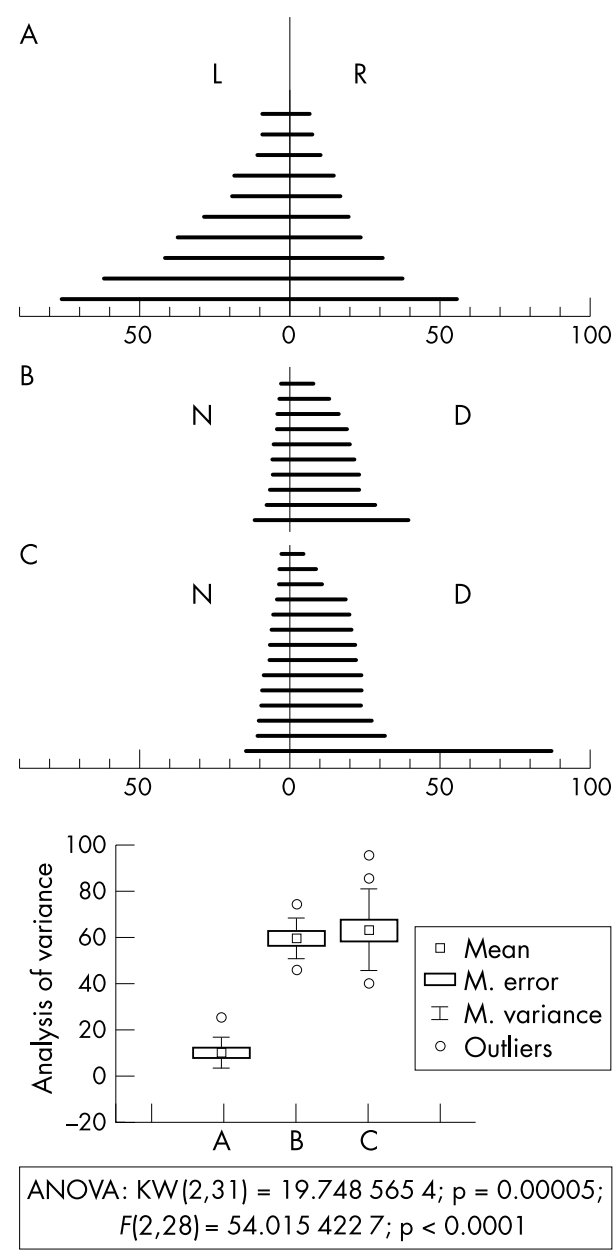

Figure 5 A: peak to peak amplitudes of the main responses in vertical EOG channels elicited by head taps on the inion in healthy subjects; $B$ : patients with unilateral vestibular dysfunction (UVD) after surgery for an acoustic neuroma; C: the heterogeneous group of patients with UVD. The bottom panel represents the asymmetry ratios of the above groups. Mean values of the EOG responses amplitudes in microvolts on both the sides were almost the same for the normal subjects (21 left (L), 23 right (R)). Means differed substantially for the patients with UVD (group B: 5.6 normal side (N), 22 lesioned side (D); group C: 5.9 healthy side, 25.6 lesioned side). Therefore, it can be said that responses consistently indicated the lesioned side for all patients. KW, Kruskall-Wallis test.

and eye behaviour have been noted ${ }^{35}$ and neural circuits controlling their movements are likely to be different. ${ }^{36}$ Both lids move synchronously in normal and pathological conditions. However, pre-motor disorders do not always affect both lids equally and if one eye is vertically displaced (for example in strabismus) the positions of the lids can be adjusted to each eye separately. ${ }^{37}$ A small but statistically significant skew torsion appears to be a permanent legacy of unilateral vestibular deafferentation and indicates an ascending type of tone imbalance of VOR in roll due to lost utricular input. ${ }^{38}{ }^{39}$ Adjustment in lid position may not accompany this recently acquired disorder unlike in patients with longstanding vertical eye displacements. However, despite distortion of the vertical eye movements unequal eye-lid interactions may even be advantageous from a diagnostic point of view, because by magnifying the inter-eye differences in movement trajectories these could facilitate the detection of even minor asymmetries in eye movements. Alternatively, the differences in tonic discharge rates of neurones driving the extraocular muscles on the left and right side due to unilateral loss of the vestibular input could lead to dif- ferences in stiffness and/or resistance of the ocular plant to vibrations.

The left and right eyes of patients with UVD, in contrast with healthy subjects, should move from a different starting position, through a different trajectory, or show a different eyeball-lid interaction on the healthy and lesioned side. Thus, if the responses were generated passively, they could reflect the otolith driven skew torsion.

\section{Brainstem reflexes}

Blink reflex

Only the trigeminofacial blink reflex shows a short latency Rl component possessing similar latency to the EOG responses in this study. This early component can generally be elicited only by stimulating the first division of the trigeminal nerve, which was avoided in this study. ${ }^{40}$ On the other hand, the later occurring symmetrical $\mathrm{R} 2$ component can be elicited by various sensory stimuli including the stimulation of sensory branches beyond the trigeminal territory. The latency of this component generally exceeds $20 \mathrm{~ms}^{41}{ }^{42}$ The R2 component is invariably accompanied by inhibition of levator muscle activity, as well as disconjugate medially and downward directed eye movement, but the orbicularis oculi response always precedes the associated eye and lid movements. ${ }^{30} 43$ Since in the present study, the responses in vertical EOG were always of higher amplitude on the side of lesioned labyrinth, even in the stage of complete facial palsy after surgery, and were absent or substantially diminished in patients of profound BVD, the responses recorded were not in any way generated by voluntary or reflex blinking.

Tonic vibration reflex of the jaw closure muscles Primary Ia spindle afferents are extremely sensitive to vibration, and thereby tonic vibration reflexes could have been elicited in certain cranial muscles in this study. Although facial muscles lack muscle spindles, the jaw closing muscles are richly endowed. The physiology of the masseter reflex has some peculiar features due to lack of presynaptic inhibition onto spindle afferents. Unlike in limb muscles, vibration not only elicits a tonic vibration reflex but also potentiates the phasic reflexes. Moreover, the jaw closure tonic vibration reflex has a unique electromyographic pattern of synchronised waves with a one to one relationship with the vibration cycles. ${ }^{44}$ EOG electrodes might naturally pick up this activity of jaw closure muscles. Nevertheless, the tonic vibration reflex evolves gradually with a rather long latency, because it depends on progressive facilitation and recruitment in polysynaptic proprioceptive pathways ${ }^{44}$ Therefore it is unlikely to appear in the first several tens of milliseconds following the single vibrational impulses applied at a low repetition rate of one to three per second, regardless of the stimulus intensity.

Proprioceptive reflexes of the extraocular muscles The ability of the oculomotor system to determine eye position is essential. According to earlier data the control of eye movement and position appeared to be primarily efferently coded. Indeed, there is no swift stretch reflex for eye muscles despite a generous complement of muscle spindles. ${ }^{45}$ Nevertheless, there is abundant evidence that the brain uses information from eye muscle proprioceptors. The palisade endings associated with the tips of the multiply innervated non-twitch muscle fibres are the most likely receptors in the principal sensory apparatus of the extraocular muscles. They are innervated by tonic motor neurones with small diameter axons, which mediate signals related only to intended eye positions. Thus they may participate in a proprioceptive system important for setting and stabilising the alignment of the eye. ${ }^{46}$ However, due to slower conduction and execution 
time the motor responses mediated by this system could be expected to occur at longer latencies as the responses in the current study.

\section{Cervico-ocular reflex}

Skull vibrations are also conducted to cervical muscles and activate their Ia afferents, thereby eliciting the cervico-ocular reflex. Electrophysiological experiments suggest that this reflex is mediated via the vestibular nuclei but the precise projection is only partially known. ${ }^{27}$ The cervico-ocular reflex is enhanced in patients with vestibular areflexia because of the increased central weighting of somatosensory neck information, which substitutes for missing vestibular input. ${ }^{47-49}$ It is likely that pathways that mediate this reflex are polysynaptic, because this reflex does not occur with latency shorter than $40 \mathrm{~ms} .^{49}$ Nevertheless, if the short latency events recorded in the current study were under the control of vestibular nuclei, the possibility of their conditioning by cervicovestibular input could not be rejected definitively.

\section{Vestibulo-ocular reflexes}

Skull as well as neck vibrations can evoke ocular movements and it is controversial whether these eye movements are caused by activation of vestibular or cervico-ocular reflexes. ${ }^{47-49}$ Since VCR with disynaptic latency can be activated by bone conducted vibrational impulses upon the skull, it is unlikely for the same stimuli not to simultaneously also elicit disynaptic VOR. Close behavioural coupling of the VCR and VOR is necessary and mammals without such coupling could not withstand natural selection pressure.

Similar eye movements to the responses recorded in the current study have been obtained by galvanic stimulation of the whole vestibular nerve, by electrical stimulation of the utricular nerve, the ampullary nerves of the semicircular canals or vestibular end organs, and by acoustic clicks as well as natural vestibular stimulation..$^{50-56}$

As a consequence of low frequency vibrations the head moves as a whole, executing parallel, or so-called translational movements to and from the site of application of vibration. Hence, the vestibular end organs embedded in the temporal bone are alternately subjected to inertial pressure. ${ }^{28}$ If the vibration pulse is realised as a sine wave, then the dominant component of the recorded events could be considered a response to a second phase of this wave probably due to the asymmetry in the otolith transfer functions. ${ }^{57} 58$

An important finding of the current study is the striking asymmetry of the responses in vertical EOG channels in the patients with UVD. All primary vestibular afferents show a marked asymmetry in bidirectional sensitivity and this nonlinearity is even more marked at the level of second order neurones. ${ }^{27}$ However, this asymmetry is more complex for the otolith system since it prefers oppositely directed hair cell deflections during dynamic translations versus static tilts and off-centre rotations.5960 Thus the enhancement of the response in vertical EOG channel on the side of vestibular insult and/or diminution on the healthy side may be a consequence of the directional asymmetry of the remaining unbalanced labyrinthine input.

Latencies of responses about 5-15 ms, with low interindividual variability in this study are in accordance with those found for the disynaptic aVOR. ${ }^{7-9}$ We have not made much effort to demonstrate the distance effect, because it was felt the technique was not suitable for detection of this kind of change. Even with more natural stimuli, due to the exponential shape of VOR response, the robust distance effect was very subtle at the onset. The rate of the gain adjustment for viewing distances of $40 \mathrm{~cm}$ versus $10 \mathrm{~cm}$ at the onset was found to differ only by one standard deviation in velocity traces using the search coil technique. ${ }^{6}$ Hence, it was easily lost in the raw EOG eye position records. On the other hand, the responses were much higher than those required for an ideal compensatory VOR and the lid artefacts could have contributed to them. Nevertheless, for high frequency stimuli used in this study, the gain of the response was primarily determined by the frequency of the stimuli, whereas some kinematical requirements of the response may have been attenuated. Apart from the vertical gaze effects on the responses that were caused by lid artefacts, the other kinematical aspects and positional effects might be consistent with otolith driven reflexes. ${ }^{18}$ The increase in amplitude of the response in vertical EOG channel on the side of adducting eye, and the decrease on the side of abducting eye, could be compatible with subsequent activation of both oblique muscles, yet evidence for disynaptic connections from otolith organs to contralateral inferior oblique muscle is lacking. ${ }^{1061}$

Finally, the apparently disconjugate responses may even reflect the basic monocular organisation of the oculomotor system. Indeed, during sleep, rapid eye movements are found to be monocular or disjunctive, and electrophysiological studies in primates have revealed that pre-motor position-vestibular-pause neurones fire in relation to monocular eye position, rather than to conjugate eye movements. ${ }^{46} 6263$

\section{CONCLUSIONS}

In the present study, EOG responses to skull vibration were generated either by phasic VOR or by passive oscillatory movements of the orbital tissues. Even if the latter were true, there was a striking asymmetry in the responses of vertical EOG channels elicited by stimuli along the naso-occipital ( $x$ ) axis in patients with UVD compared with normal subjects. This finding was at least in accordance with the movement of the eyes on the healthy and lesioned side from different starting positions, due to otolith mediated sustained skew torsion in patients with UVD. This asymmetry provides a consistent, longlasting lateralising clue for testing at least complete UVD. We are aware of several drawbacks of our study due to the limited reliability of the methods of recording and analysis. However, despite these limitations the findings provide at least circumstantial evidence for the possibility of investigating VOR using vibrational impulse stimuli.

\section{ACKNOWLEDGEMENTS}

We express our thanks to professors M Drobný and L Lisý for their intellectual support and Dr M Kamenický for expert assistance.

\section{Authors' affiliations}

P Jombik, Zvolen Hospital, Department of Neurology, Laboratory of Clinical Neurophysiology, Zvolen, Slovak Republic

V Bahýl, Technical University in Zvolen, Faculty of Wood Sciences and Technology, Department of Physics and Applied Mechanics, Zvolen, Slovak Republic

Competing interests: none declared

\section{REFERENCES}

1 Young ED, Fernández C, Goldberg JM. Responses of the squirrel monkey vestibular neurons to audio-frequency sound and vibration. Acta Otolaryngol (Stockh) 1977;84:352-60.

2 Fernández C, Goldberg JM. Physiology of peripheral neurons innervating otolith organs of the squirrel monkey. III. Response dynamics. J Neurophysiol 1976;39:996-1008

3 Paige GD, Telford L, Seidman SH, et al. Human vestibulo-ocular reflex and its interactions with vision and fixation distance during linear and angular head movement. J Neurophysiol 1998;80:2391-404.

4 Telford L, Seidman SH, Paige GD. Canal-otolith interactions in the squissel monkey vestibulo-ocular reflex and the influence of fixation distance. Exp Brain Res 1998;118:115-25.

5 Paige GD, Tomko DL. Eye movement responses to linear head motion in the squirrel monkey. II. Visual-vestibular interactions and kinematic considerations. J Neurophysiol 1991;65:1183-96. 
6 Angelaki DE, McHenry $M Q$. Short-latency primate vestibuloocular responses during translation. J Neurophysiol 1999;82:1651-4.

7 Maas EF, Heubner WP, Seidman SH, et al. Behavior of the human horizontal vestibulo-ocular reflex in response to high-acceleration stimuli. Brain Res 1989:499:153-6.

8 Collewijn H, Smeets JBJ. Early components of the human vestibulo-ocular response to head rotation: latency and gain. I Neurophysiol 2000;84:376-89.

9 Crane BT, Demer JL. Human horizontal vestibulo-ocular reflex initiation: effects of acceleration, target distance, and unilateral deafferentation. J Neurophysiol 1998;80:1151-66.

10 Uchino $Y$, Sasaki $M$, Sato $H$, et al. Utriculoocular reflex arc of the cat. J Neurophysiol 1996;76:1896-903.

11 Bush GA, Miles FA. Short-latency compensatory eye movements associated with a brief period of free fall. Exp Brain Res 1996;108:337-40.

12 Gianna CC, Gresty MA, Bronstein AM. The human linear vestibulo-ocular reflex to transient accelerations: visual modulation of suppression and enhancement. J Vestibular Res 2000;10:227-38.

13 Crane BT, Tian J, Wiest G, et al. Initiation of the human heave linear vestibuloocular reflex. Exp Brain Res 2003;148:247-55.

14 Uchino $Y$, Ikegami H, Sasaki $M$, et al. Monosynaptic and disynaptic connections in the utriculo-ocular reflex arc of the cat. J Neurophysiol 1994;71:950-8.

15 Raphan T, Cohen B. The vestibulo-ocular reflex in three dimensions. Exp Brain Res 2002; 145:1-27.

16 Telford L, Seidman SH, Paige GD. Dynamics of the squirrel monkey linear vestibulo-ocular reflex and interaction with fixation distance. J Neurophysio 1997:78:1775-90.

17 Telford L, Seidman SH, Paige GD, et al. Dynamics of squirrel monkey linear vestibulo-ocular reflex and interactions with fixation distance. Exp Brain Res 1998;118:115-25.

18 Halmagyi GM, Yavor RA, Colebatch JG. Tapping the head activates the vestibular system: a new use for the clinical reflex hammer. Neurology 1995;45:1927-9.

19 Sheykholeslami K, Murofushi T, Habibi Kermani M, et al. Bone-conducted evoked myogenic potentials from the sternocleidomastoid muscle. Acta Otolaryngol 2000;120:731-4.

20 Welgampola MS, Rosengren SM, Halmagyi GM et al. Vestibular activation by bone conducted sound. J Neurol Neurosurg Psychiatry 2003;74:771-8.

21 Hamann KF, Schuster EM. Vibration-induced nystagmus - a sign of unilateral vestibular deficit. ORL J Otorhinolaryngol Relat Spec 1999;61:74-79.

22 Michel J, Dumas G, Lavieille JP, et al. Diagnostic value of vibration-induced nystagmus obtained by combined vibratory stimulation applied to the neck muscles and skull of 300 vertiginous patients. Rev Laryngol Otol Rhinol, 2001;122, 2:89-94

23 Karlberg M, Aw ST, Halmagyi GM, et al. Vibration-induced shift of the subjective visual horizontal. A sign of unilateral vestibular deficit. Arch Otolaryngol Head Neck Surg 2002;128:21-7.

24 Karlberg M, Aw ST, Black RA, et al. Vibration-induced ocular torsion and nystagmus after unilateral vestibular deafferentation. Brain 2003; 126:956-964.

25 Pyykkö I, Aalto H, Grönfors T, et al. Vestibular evoked responses in man: Methodological aspects. Acta Otolaryngol (Stockh) 1995;520(suppl): 1 17-19.

26 Zou J, Bretlau P, Pyykkö I, et al. Sensorineural hearing loss after vibration: an animal model for evaluating prevention and treatment of inner ear hearing loss. Acta Otolaryngol 2001;121:143-8.

27 Baloh RW, Honrubia V. Clinical neurophysiology of the vestibular system. Oxford: Oxford University Press, 2001.

28 Hood JD. Bone conduction: a review of present position with especial reference to the contributions of Dr Georg von Békésy. J Acoust Soc Am 1962;34:1325-32.

29 Berthoz A, Rousié D. Physiopathology of otolith-dependent vertigo Contribution of the cerebral cortex and consequences of cranio-facial asymmetries. In: Tran Ba Huy P, Toupet $M$, eds. Otolith functions and disorders. Adv Otorhinolaryngol Basel, Karger, 2001;58:48-67.

30 Collewijn H, Van der Steen J, Steinman RM. Human eye movements associated with blinks and prolonged eyelid closure. J Neurophysiol 1985;54:11-27.

31 Lueck CJ, Kennard C. Oculography and techniques for eye movement recording. In:Daroff R, Neetens A.eds. Neurological organization of ocular movement. Berkeley: Kugler and Ghedini, 1990:485-502.

32 Vercher J-L, Gauthier GM, Marchetti E, et al. Origin of eye movements induced by high frequency rotation of the head. Aviat Space Environm Med 1984;55:1046-50.

33 Peli E, García-Pérez MA. Motion perception during involuntary eye vibration Exp Brain Res 2003;149:431-8.

34 García-Pérez MA, Peli E. Simple non-invasive measurement of rapid eye vibration. J Sound Vibr 2003;262:877-88.
35 Fuchs AF, Becker W, Ling L, et al. Discharge patterns of levator palpebrae superioris motoneurons during vertical lid and eye movements in the monkey. J Neurophysiol 1992;68:233-43.

36 Chen B, May PJ. Premotor circuits controlling eyelid movements in conjunction with vertical saccades in the cat: I. The rostral interstitial nucleus of the medial longitudinal fasciculus. J Comp Neurol 2002;450:183-202.

37 Schmidtke K, Büttner-Ennever JA. Nervous control of eyelid function. A review of clinical experimental and pathological data. Brain 1992;1 15:227-47.

38 Curthoys IS, Dai MJ, Halmagyi GM. Human ocular torsional position before and after unilateral vestibular neurectomy. Exp Brain Res 1991:85:218-225.

39 Brandt T, Dietrich M. Central vestibular syndromes in roll, pitch, and yaw planes: topographic diagnosis of brainstem disorders. Neuroophthalmology 1995; 15:291-303.

40 Kimura J. The blink reflex as a clinical test. In: Aminoff MJ, eds. Electrodiagnosis in clinical neurology. New York: Churchill Livingstone, 1986:347-83.

41 Rushworth G. Observation on blink reflexes. J Neurol Neurosurg Psychiatry 1962;25:93-108.

42 Mirwa H, Imamura N, Kogahara K, et al. Somatosensory evoked blink response: findings in patients with Miller Fisher syndrome and in normal subjects. J Neurol Neurosurg Psychiatry 1995;58:95-9.

43 Bour L, Aramideh M, Ongerboer de Visser BW. Neurophysiological aspects of eye and eyelid movements during blinking in humans. J Neurophysiol 2000:83:166-176.

44 Desmedt JE. Mechanisms of vibration-induced inhibition or potentiation: tonic vibration reflex and vibration paradox in man. In: Desmedt JE, eds. Advances in neurology. Vol. 39. Motor control in health and disease. New York: Raven Press, 1983:671-83.

45 Ruff R, Kaminski $H$, Maas $E$, et al. Ocular muscles: physiology and structurefunction correlations. In: Daroff R, Neetens A, eds. Neurological organization of ocular movement. Berkeley: Kugler and Ghedini, 1990:331-52.

46 Büttner-Ennever JA, Horn AKE. The neuroanatomical basis of oculomotor disorders: the dual motor control of extraocular muscles and its possible role in proprioception. Curr Opin Neurol 2002;15:35-43.

47 Yagi T, Ohyama Y. Three-dimensional analysis of nystagmus induced by neck vibration. Acta Otolaryngol (Stockh) 1996;116:167-9.

48 Strupp M, Arbusov V, Dietrich M, et al. Perceptual and oculomotor effects of neck muscle vibration in vestibular neuritis. Ipsilateral somatosensory substitution of vestibular function. Brain 1998;121:677-85.

49 Popov KE, Lekhel H, Faldon $M$, et al. Visual and oculomotor responses induced by neck vibration in normal subjects and labyrinthine-defective patients. Exp Brain Res 1999;128:343-52.

50 Séverac Cauquil A, Faldon M, Popov K, et al. Short latency eye movements evoked by near-threshold galvanic vestibular stimulation. Exp Brain Res 2003;148:414-18.

51 Suzuki J-I, Tokumasu K, Goto K. Eye movements from single utricular nerve stimulation in the cat. Acta Otolaryngol (Stockh) 1969:68:350-62.

52 Cohen B, Suzuki J-I, Bender MB. Eye movements from semicircular canal nerve stimulation in the cat. Am Otol Rhinol Laryngol 1964;73:153-69.

53 Flur E, Mellström A. Utricular stimulation and oculomotor reactions. Laryngoscope 1970;80:1701-12.

54 Flur E, Mellström A. Saccular stimulation and oculomotor reactions. Laryngoscope 1970;80:1713-21

55 Halmagyi GM, McGarvie LA, Aw ST, et al. The click-evoked vestibulo-ocular reflex in superior semicircular canal dehiscence. Neurology 2003;60:1172-5.

56 Diamond SG, Markham CH. Ocular counterrolling as an indicator of vestibular otolith function. Neurology 1983:1460-9.

57 Halswanter T, Jaeger R, Mayr S, et al. Three-dimensional eye-movement responses to off-vertical axis rotations in humans. Exp Brain Res 2000;134:96-106.

58 Courthoys LS, Halswanter T, Black RA, et al. Off-center yaw rotation: effect of naso-occipital linear acceleration on the nystagmus response of normal human subjects and patients after unilateral vestibular loss. Exp Brain Res 1998; 123:425-38

59 Day MJ, Curthoys IS, Halmagyi GM. Perception of linear acceleration in the roll plane before and after unilateral vestibular neurectomy. Exp Brain Res 1989;77:315-28

60 Lempert T, Gianna C, Brookes G, et al. Horizontal otolith-ocular responses in humans after unilateral vestibular deafferentation. Exp Bran Res 1998; 118:533-40.

61 Isu N, Graf W, Sato H, et al. Sacculo-ocular reflex connectivity in cats. Exp Brain Res 2000;131:262-8.

62 Zhou W, King WM. Binocular eye movements not coordinated during REM sleep. Exp Brain Res 1997;117:153-60.

63 McConville K, Tomlinson RD, King WM, et al. Eye position signals in the vestibular nuclei: consequences for models of integrator function. J Vestib Res 1994;4:391-400 


\section{PostScript}

\section{LETTERS}

\section{A case of late onset sporadic Parkinson's disease with an A53T mutation in $\alpha$-synuclein}

Parkinson's disease is a common progressive neurological disorder characterised by loss of nigral dopaminergic neurones. Rare autosomal dominant familial cases have been associated with point mutations in $\alpha$-synuclein, ${ }^{12}$ but the vast majority of cases occur sporadically in older patients without an obvious cause. We now report a unique case of typical late onset Parkinson's disease without a family history which was associated with an A53T mutation in $\alpha$-synuclein.

\section{Case report}

A war veteran of Polish origin was initially referred for assessment of his parkinsonian condition in 1997 at the age of 74. At presentation his history was of progressive bradykinesia, difficulty in rising from his chair, a tendency to fall, and mild tremor. He was a smoker and had been treated for hypertension and hypercholesterolaemia, but he gave no clear history of cerebrovascular disease. His Austrian mother died at 91 years of age and his French father lived to 89 , neither suffering from symptoms of Parkinson's disease. He had four brothers and three sisters, none of whom had symptoms of Parkinson's disease (two died in their 20 s during the war, the others died at ages $68,76,78$, and 86 , and one has lost touch with the family). Furthermore, his three children, each now in their sixth decade, currently have no diagnosis of Parkinson's disease. The family know of no relatives of Italian or Greek origin.

Examination was consistent with Parkinson's disease, with a typical shuffling gait, bilateral cogwheel rigidity, and mild tremor, but no pyramidal or cerebellar signs. Investigations were normal, but magnetic resonance imaging of his brain was not possible because of metal shrapnel in his left orbit, face, and nose from the second world war. Computed tomography of the brain showed only mild age related cerebral atrophy without evidence of vascular disease.

Before presentation he had been prescribed co-beneldopa $62.5 \mathrm{mg}$ three times a day with some symptomatic benefit. In July 1997 a five week trial off levodopa caused a deterioration in his symptoms, therefore his co-beneldopa was restarted and increased to $125 \mathrm{mg}$ three times daily, and selegiline was started. On this treatment his symptoms remained stable for the next three years, and a second trial without levodopa or a dopamine agonist was attempted in May 2000, which again provoked marked bradykinesia and deterioration in his gait. His treatment was restarted after only five days, following which the symptoms once again resolved, showing the clear levodopa responsive element to his condition. He died in August 2002 but a necropsy examination of the brain was not undertaken.

\section{Genetic analysis}

PCR primers were designed from 5' untranslated region (UTR) and 3'UTR spanning each exon of $\alpha$-synuclein (from NACP/ $\alpha$-synuclein sequences submitted to NCBI database; accession number U46896-U46901; primer pairs designed to amplify exon 3 were $3 \mathrm{~F}$ : GAGGACCTCCTGTTAGCTGG, and 3R: GACT GATATGTTCTTAGATGCTC. Polymerase chain reaction (PCR) products were purified using QIAquick columns and sequenced according to the manufacturer's protocols by dye terminator (BigDye) methods using an ABI 377 automated sequencer (Applied Biosystems, Foster City, California, USA). All sequences were edited and confirmed by entering them into the BLAST algorithm database at the National Center for Biotechnology Information (http://www.ncbi.nlm.nih.gov/blast).

A single mutation from $G$ to $A$ was found at nucleotide 209 in exon 3 of the $\alpha$-synuclein gene from this patient (fig 1A). The sample was reanalysed twice following this observation, and also verified in the reverse direction. Restriction digest of the PCR products was carried out with Tsp45 I (New England BioLabs, Beverly, Massachusetts, USA), and digested products were separated by electrophoresis on a $2 \%$ agarose gel (fig $1 \mathrm{~B}$ ). The results correspond to an alanine to threonine shift at position 53 of $\alpha$-synuclein (an A53T mutation).

\section{Comment}

The discovery of families with autosomal dominant Parkinson's disease together with the subsequent development of symptomatic $\alpha$-synuclein transgenic models of the disease ${ }^{3}$ has provided strong support that a point mutation in $\alpha$-synuclein is sufficient to cause this disorder. Furthermore, the discovery of $\alpha$-synuclein in the Lewy bodies of patients with the common sporadic form of Parkinson's disease ${ }^{4}$ has suggested that this protein may well play a central role in all forms of the disease.

Until now the A53T mutation in $\alpha$-synuclein has only been described in a large kindred of Italian origin and a small number of unrelated families of Greek origin, each with autosomal dominant inheritance of the Parkinson's disease phenotype. ${ }^{1}$ The clinical phenotype and response to levodopa in the Italian kindred is relatively typical for Parkinson's disease, but with an earlier mean age of onset at 46 (SD 13) years, and a relatively rapid course, averaging 9.7 years from onset to death. ${ }^{5}$

The case we describe had no such family history, first noticed symptoms in his eighth decade, and had a clinical phenotype compatible with sporadic idiopathic Parkinson's disease. He had a relatively mild tremor, as noted in the Italian kindred. ${ }^{5}$ This case is therefore of particular interest as it appears to represent a unique sporadic mutation in $\alpha$ synuclein that was not found in the remaining 60 control and patient samples that we analysed, and has not been reported in other series. Furthermore, despite carrying the
A

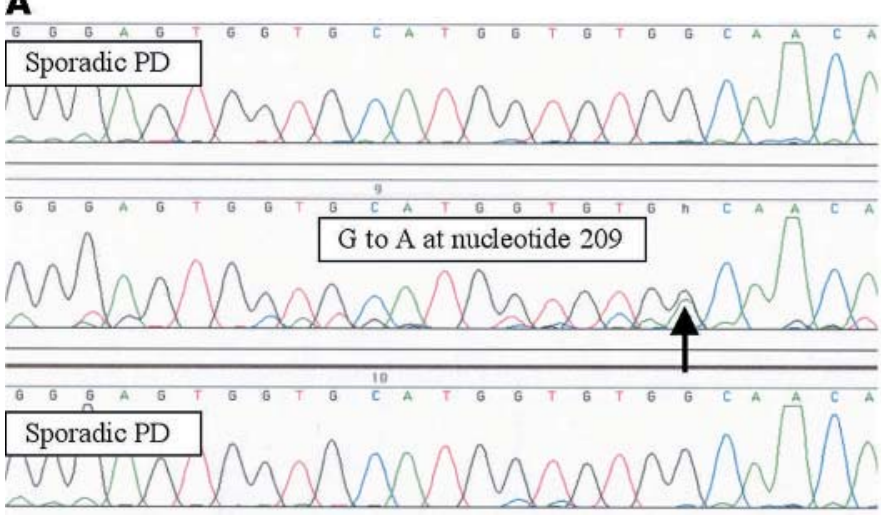

B

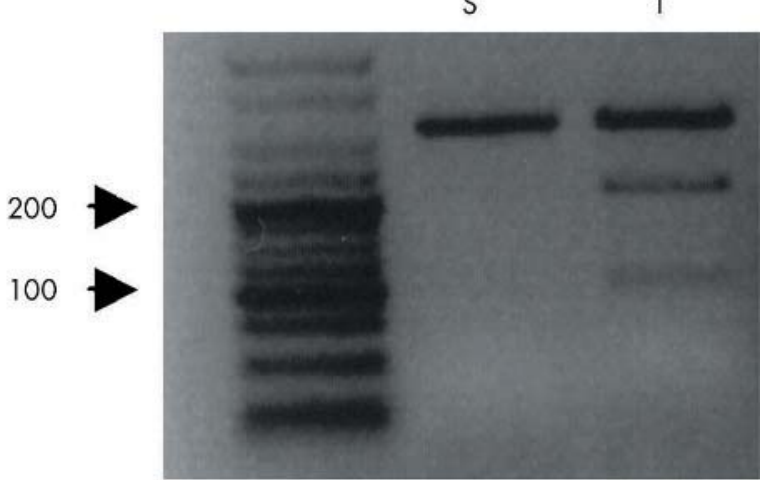

Figure 1 (A) Electropherogram of three patients with Parkinson's disease, the middle showing the mutation at nucleotide G209A in exon 3. Results were confirmed in the opposite direction. (B) Restriction digest of exon 3 polymerase chain reaction product. The patient is heterozygous for the $G$ to $A$ mutation, thus creating a new recognition site for Tsp45 I which cleaves the wild type 393 bp product to two fragments of 258 bp and 135 bp (S, sample from patient with sporadic Parkinson's disease; I, sample from index case). 
genetic mutation this patient developed symptoms much later in life than most members of the Italian kindred described above.

The penetrance of the A53T $\alpha$-synuclein gene in the Italian kindred has been estimated at $85 \%^{1}$ so, although unlikely, it is theoretically possible that in this case the mutated gene is asymptomatic and the patient has developed unrelated late onset sporadic Parkinson's disease. Alternatively it is possible that the relatively mild late onset of Parkinson's disease in this patient represents a dose effect of the mutant gene. For example, both duplication and triplication of the $\alpha$-synuclein gene locus have recently been found to cause familial Parkinson's disease, ${ }^{67}$ and the different severity of clinical phenotype seems to be correlated with the dose of $\alpha$-synuclein.

This unique case extends the repertoire of patients in whom Parkinson's disease is associated with point mutations of $\alpha$-synuclein. While we do not advocate routine clinical screening, this case suggests that further evaluation of mutations on this gene should be considered in cases of sporadic Parkinson's disease.

\section{Acknowledgements}

Our work is supported by the MRC, the Parkinson's Disease Society, and the Raymond and Beverly Sackler Fund.

A W Michell, R A Barker, R Raha-Chowdhury Cambridge Centre for Brain Repair, Cambridge, UK

S K Raha

Department of Integrated Medicine, Princess of Wales Hospital, Bridgend, UK

Correspondence to: Dr Andrew Michell, Cambridge Centre for Brain Repair, Forvie Site, Robinson Way, Cambridge CB2 2PY, UK; awm13@cam.ac.uk

doi: 10.1136/jnnp.2004.046425

Competing interests: none declared

\section{References}

1 Polymeropoulos MH, Lavedan C, Leroy E, et al. Mutation in the alpha-synuclein gene identified in families with Parkinson's disease. Science 1997; 276:2045-7.

2 Kruger R, Kuhn W, Muller T, et al. Ala30Pro mutation in the gene encoding alpha-synuclein in Parkinson's disease. Nat Genet 1998;18:106-8.

3 Feany MB, Bender WW. A Drosophila model of Parkinson's disease. Nature 2000;404:394-8.

4 Spillantini MG, Schmidt ML, Lee VM, et al. Alphasynuclein in Lewy bodies. Nature

1997;388:839-40.
5 Golbe LI, Di lorio G, Bonavita $\mathrm{V}$, et al. A large kindred with autosomal dominant Parkinson's disease. Ann Neurol 1990;27:276-82.

6 Chartier-Harlin MC, Kachergus J, Roumier C, et al. Alpha-synuclein locus duplication as a cause of familial Parkinson's disease. Lancet 2004;364:1 167-9.

7 Singleton $A B$, Farrer $M$, Johnson J, et al. alphaSynuclein locus triplication causes Parkinson's disease. Science 2003;302:841.

\section{Decreased CSF hypocretin-1 (orexin-A) after acute haemorrhagic brain injury}

The novel hypothalamic neuropeptides orexins, or hypocretins, have gained much attention as potent modulators of various different physiological functions. ${ }^{1}$ Deficient orexin neurotransmission may be responsible for excessive somnolence, as shown in several conditions related to secondary narcolepsy, through direct or indirect damage to the posterior hypothalamus and its connections. ${ }^{2}$ We have studied for the first time the longitudinal changes of hypocretin-1 concentrations in cerebrospinal fluid (CSF) in patients with acute haemorrhagic brain injury.

Nine patients from a previously reported cohort $^{3}$ and 21 controls (seven women and five men, median age 38 years, range 17 to 70) with other neurological disease were enrolled in the study (table 1). The patient group included five subjects with intracerebral haemorrhage and five with subarachnoid haemorrhage (table 1). All patients had extraventricular drains inserted within a median of two days of disease onset (range 2 to 36 ) as a treatment procedure because of increasing signs of hydrocephalus. Morning CSF samples were collected twice: first between day 1 and day 2 after catheter insertion, and second between day 4 and day 10. Patients were assessed using the Glasgow coma score (GCS) at presentation and the Glasgow outcome score (GOS) after three months.

The control group consisted of two patients with primary dementia, two with chronic headache, one with ataxia syndrome, and seven with non-specific neurological symptoms. None reported sleep abnormalities.

All samples were stored at $-80^{\circ} \mathrm{C}$ until analysis. Hypocretin- 1 was measured blind to diagnosis by direct radioimmunoassay of $100 \mu \mathrm{l}$ of CSF (Phoenix Pharmaceuticals, Belmont, California, USA; detection limit 40 $\mathrm{pg} / \mathrm{ml}$, intra-assay variation $<5 \%$ ), as described previously. ${ }^{4}$ Samples with undetectable concentrations (value below $40 \mathrm{pg}$ / $\mathrm{ml}$ ) were operationally plotted at $0 \mathrm{pg} / \mathrm{ml}$. Statistical tests were carried out with the
GraphPad InStat 3.05 software package using the non-parametric Mann-Whitney U test.

There was a significant difference in median CSF hypocretin-1 concentrations between the controls (319.4 (302 to 361) $\mathrm{pg} / \mathrm{ml}$ ) and acute brain injury patients (100.4 (0 to 145.2) $\mathrm{pg} / \mathrm{ml}$ ) (calculated from the means of two measurements; median (range)). No difference was found for sex or age (table 1). The concentration of hypocretin-1 in CSF on the first day of sample collection (24 hours after catheter insertion) was 98.8 (0 to 147) pg/ml and did not change significantly over the observation period ( 114.0 (0 to 144) pg/ml). All concentrations were either lower than control values $(>200$ $\mathrm{pg} / \mathrm{ml}$ ), in the intermediate range ( 110 to 200 $\mathrm{pg} / \mathrm{ml})$, or in the very low, narcolepsy range $(<110 \mathrm{pg} / \mathrm{ml}) .{ }^{4}$ Two patients (Nos 4 and 6) had undetectable levels, while all others had moderately decreased values compared with the cut off level of $196 \mathrm{pg} / \mathrm{ml}$. In patient 6 , the hypocretin-1 level increased to $53 \mathrm{pg} / \mathrm{ml}$, but it remained undetectable in patient 4 . Both patients suffered from spontaneous intracerebral haemorrhage, which was localised either to the thalamus (patient 4 ) or to the midbrain (patient 6). Other patients with moderately decreased hypocretin-1 levels were diagnosed as having subarachnoid haemorrhage (six patients) and intracerebral haemorrhage in the frontal lobe (one patient). There was no correlation between hypocretin-1 levels and GCS at disease onset or GOS at three months after disease onset,

\section{Comment}

This is the first study to show decreased levels of hypocretin-1 in the CSF of patients in the first week after acute brain injury caused by haemorrhagic stroke. Our data are in line with previous observations in patients with traumatic brain injury.

The findings seem important in the light of a study investigating long term outcome in patients after subarachnoid haemorrhage. ${ }^{2}$ More than $75 \%$ of patients reported excessive fatigue or daytime sleepiness, which persisted for long period (months to years) after the event. However, the exact mechanism responsible for the complaints remained unknown. Although the aetiology may be quite complex, an abnormality in the hypocretin/orexin system could make an important contribution to the phenomenon.

How intracerebral haemorrhage affects hypothalamic function remains obscure. In two of our patients direct damage to the thalamic/brain stem circuits appeared to be responsible. However, the remainder of the patients had a subarachnoid bleed or lesions in remote brain structures unrelated to the

Table 1 Patient characteristics

\begin{tabular}{|c|c|c|c|c|c|c|c|}
\hline Case & Sex & Age (y) & Diagnosis & Lesion and complications & GCS & IVS & GOS \\
\hline 1 & M & 45 & $\mathrm{ICH}$ & $\mathrm{HC}$ & $6 / 15$ & 2 & 24 \\
\hline 2 & M & 66 & SAH (ACA aneurysm) & $\mathrm{HC}$, multiple infarcts & $5 / 15$ & 2 & 21 \\
\hline 3 & M & 54 & SAH (PCA aneurysm) & $\mathrm{HC}$, occipital infarct, re-bleed & $3 / 15$ & 15 & 151 \\
\hline 4 & $\mathrm{~F}$ & 59 & $\mathrm{ICH}$ (spontaneous) & $\mathrm{HC}$, thalamus & $9 / 15$ & 4 & 41 \\
\hline 5 & M & 59 & SAH & $\mathrm{HC}$ & $14 / 15$ & 2 & 21 \\
\hline 6 & $\mathrm{~F}$ & 59 & $\mathrm{ICH}$ (spontaneous) & HC, midbrain, re-bleed & $6 / 15$ & 2 & 21 \\
\hline 7 & M & 72 & SAH & $\mathrm{HC}$ & $14 / 15$ & 2 & 24 \\
\hline 8 & $\mathrm{~F}$ & 70 & SAH (ACA aneurysm) & $\mathrm{HC}$, re-bleed & $15 / 15$ & 8 & 85 \\
\hline 9 & M & 45 & $\mathrm{ICH}$ (spontaneous) & Frontal lobe & $8 / 15$ & 36 & 363 \\
\hline
\end{tabular}

ACA, anterior cerebral artery; F, female; GCS, Glasgow coma scale (on admission); GOS, Glasgow outcome scale (after 3 months); HC, hydrocephalus; ICH, intracranial haemorrhage; IVS, intraventricular shunt (days after disease onset); $M$, male; PCA, posterior cerebral artery; SAH, subarachnoid haemorrhage; $y$, years. 
hypothalamus. Previously we provided evidence of diffuse axonal injury in these patients $s^{5}$ and speculated that this represented one mechanism of disruption of the hypothalamic circuits. A remote chemical mechanism related to the presence of blood might also contribute to the decrease in hypocretin-1 levels in the CSF. It is well recognised that a large amount of blood entering the CSF compartment or brain parenchyma produces neurotoxic effects through various different mechanisms, including oxidative haem and iron metabolism and secondary oedema with abnormalities of brain perfusion. ${ }^{6}$

The decreased hypocretin-l concentrations in our patients might have resulted from the dilution effect of the bleed into the CSF with the development of secondary hydrocephalus in the course of their disease. However, this seems rather unlikely as intraventricular drainage was initiated early and the first samples were collected at least 24 hours after catheter insertion. One might also expect that in hydrocephalus complicating subarachnoid haemorrhage there would be an accumulation of CSF constituents owing to reduced absorption by subarachnoid villi, which would go against our hypothesis; however, an opposite effect was observed and this persisted during the course of the disease.

An important caveat of our study is that control samples were obtained by lumbar puncture, and lumbar CSF is likely to have a different composition from cisternal CSF. However, the concentrations of different neurotransmitters in the ventricular CSF are reported to be higher than in corresponding lumbar puncture specimens. ${ }^{3}$

Further studies are needed to investigate prospectively the relation between hypocretin-1 production and sleep-wake cycle abnormalities in patients after haemorrhagic stroke.

K Rejdak

Department of Neurology, Medical University, Lublin Poland

A Petzold

Department of Neuroinflammation, Institute of Neurology, Queen Square, London WCI, UK

Center for Narcolepsy, Stanford

L Lin California, USA

M Smith

Neurosurgical Intensive Care Unit, National Hospital for Neurology and Neurosurgery, Queen Square

N Kitchen

Victor Horsley Department of Neurosurgery, The National Hospital for Neurology and Neurosurgery,

Queen Square
E J Thompson

Department of Neuroinflammation, Institute of Neurology, Queen Square, London, UK

Correspondence to: Dr K Rejdak, Department of Neurology, Medical University of Lublin, 8 Jaczewskiego Street, 20-954 Lublin, Poland;

krejdak@europe.com

doi: 10.1136/jnnp.2004.049247

Competing interests: none declared

\section{REFERENCES}

1 Nishino S. The hypocretin/orexin system in health and disease. Biol Psychiatry 2003;54:87-95.

2 Ogden JA, Utley T, Mee EW. Neurological and psychosocial outcome 4 to 7 years after subarachnoid hemorrhage. Neurosurgery 1997:41:25-34.

3 Petzold A, Rejdak K, Belli A, et al. Axonal pathology in subarachnoid and intracerebral hemorrhage. J Neurotrauma (in press).

4 Ripley B, Overeem S, Fujiki N, et al. CSF hypocretin/orexin levels in narcolepsy and other neurological conditions. Neurology

2001;57:2253-8.

5 Reiber H. Dynamics of brain derived proteins in cerebrospinal fluid. Clin Chim Acta 2001;310:173-86.

6 Macdonald RL, Weir BK. A review of hemoglobin and the pathogenesis of cerebral vasospasm Stroke 1991;22:971-82.

\section{BOOK REVIEW}

\section{The essential handbook of memory disorders for clinicians}

Edited by Alan D Baddeley, Michael D Kopelman, Barbara A Wilson. Published by John Wiley \& Sons Ltd, 2004, pp 392, £26.99 (paperback). ISBN 0-470-09141-X.

Any reader who is familiar with the editors' comprehensive and authoritative "Handbook of Memory Disorders" will experience a powerful sense of déjà vu on opening this volume, and may be forgiven for wondering what exactly the point of it is. The Readers' Digest edition? Neuropsychology lite? With its 392 pages this is hardly a pocket companion. The real reason can be found in an (extremely brief) preface: the 35 chapters of the original work had resulted in a "heftier and more expensive book, which might well be seen as less directly relevant to clinical practice". In other words, a self-confessed case of "mega biblion mega kakon", and doubtless a publisher's marketing wheeze.

A wizard one? Perhaps. Thirty-five chapters have been whittled down to 15, of which all but one have direct clinical relevance, ranging from the amnesias of childhood to a review of rehabilitative strategies for the memory impaired. The exception is Baddeley's opening essay on contemporary and historical views of the psychology of memory. While such an overview is by no means out of place, its theoretical emphasis might perhaps have been acknowledged by according it the title and status of Introduction rather than merely "Chapter 1".

The remaining contents are also rather arbitrarily ordered, and the clinician in search of an up-to-the-minute review of some aspect of diagnosis or management is hardly guided by it to the most relevant pages. All the important themes-evaluation, differential diagnosis, management-are well represented, but needlessly interleaved. Chapters dealing with the assessment of memory disorders and the distinction between disorders of memory and other cognitive systems come after those in which specific subtypes of memory dysfunction are discussed. No fewer than four chapters discuss remediation and rehabilitation, while the discussion of retrograde amnesia is entirely subsumed within a review of psychogenic disorders, and the critically important topic of semantic memory is completely neglected.

So while this handbook will undoubtedly be of interest to clinicians, I suspect that many will prefer to distil the essence of the subject from the more comprehensive parent volume, and regard the additional heft and cost as a price worth paying.

P Garrard

\section{CORRECTION}

doi: 10.1136/jnnp.2003.027615corr 1

Jombik $\mathrm{P}$ and Bahyl V. Short latency responses in the averaged electro-oculogram elicited by vibrational impulse stimuli applied to the skull: could they reflect vestibuloocular reflex function? (J Neurol Neurosurg Psychiatry 2005;76:222-8). The first sentence of figure l legend should read: Averaged electro-oculography (EOG) responses in a normal subject elicited by stimuli along the interaural axis. 